das Gebiet des Familienrechts und hat hier gleichfalls wichtige und wegweisende Entscheidungen vorbereitet. Erwähnenswert sind insbesondere der Beschluss des Ersten Senats zum Sorgerechtsverlust der Mutter bei Ehelichkeitserklärung des Kindes ${ }^{13}$ sowie der Beschluss zur Bestimmung des Familiennamens bei Eheschließungen ${ }^{14}$. Beide Beschlüsse waren Anstoß für sehr grundlegende Änderungen des Familien- und $\mathrm{Na}$ mensrechts und veranlassten den Gesetzgeber, wenn auch zum Teil nur sukzessive und nur nach erneuter Entscheidung des Bundesverfassungsgerichts, das Recht maßgeblich zu ändern. Der Beschluss zum Sorgerechtsverlust der Mutter bei Ehelichkeitserklärung des Kindes war der entscheidende Anstoß für die Erarbeitung eines Gesetzentwurfs, der auch nicht verheirateten Eltern generell die Möglichkeit einer gemeinsamen Sorge für die Kinder einräumt. Beim Namensrecht führte die von Helga Seibert vorbereitete Entscheidung zunächst einmal dazu, dass die Frau das Recht erhielt, ihren Mädchennamen beizubehalten. Es bedurfte dann über Jahre versetzt weiterer - letztlich vom Bundesverfassungsgericht erzwungener - Ge- setzesänderungen bis zum heutigen Recht, das jedem Ehepartner die Beibehaltung seines Namens ermöglicht.

Diese drei großen Richterpersönlichkeiten, die bekanntermaßen von Statur her zum Teil gar nicht groß, sondern zierlich und klein waren, sind für uns hier im Bundesverfassungsgericht heute - wie auch viele andere - immer noch Vorbild und Ansporn. Ich bin mir sicher, dass es Vielen hier im Raume ebenso geht.

In diesem Sinne möchte ich Sie anspornen und ermutigen, Ihren rechtspolitischen Erfolgskurs fortzusetzen; ich wünsche Ihnen zunächst einmal interessante und spannende Referate, offene und kontroverse Diskussionen und vor allem neue Erkenntnisse, die möglicherweise auch einmal die Rechtsprechung des Bundesverfassungsgerichts beeinflussen werden. Ich wünsche Ihnen ein gutes Gelingen des 38. Kongresses des Deutschen Juristinnenbundes.

\footnotetext{
13 BVerfGE 84, 168.
}

14 BVerfGE 84, 9 ff.

\title{
Wir müssen auch in Zukunft eine Menge tun
}

\section{Brigitte Zypries MdB}

Bundesministerin der Justiz, Berlin/Darmstadt

Sehr geehrter Herr Präsident des Bundsverfassungsgerichts, sehr geehrte Damen und Herren, liebe Jutta Wagner,

ich bin gerne zu Ihnen gekommen, denn Sie haben sich ein besonders wichtiges Thema vorgenommen: Die bessere Integration von Migrantinnen ist eine enorme Herausforderung für unsere Gesellschaft und ich bin sehr dankbar, dass sich der Juristinnenbund dieser Aufgabe mit soviel Engagement annimmt. Wie brisant die "Integration durch Recht" ist, liegt auf der Hand. Ich erinnere nur an die Reform des Staatsangehörigkeitsrechts, an das Zuwanderungsgesetz und an die politischen Kampagnen, die es gegen diese Projekte gegeben hat. Für Rot-Grün ging es damals darum, gesellschaftliche Realität und Recht wieder stärker miteinander in Einklang zu bringen. Mit den Reformgesetzen haben wir erstmals anerkannt, dass Deutschland ein Einwanderungsland ist. Und wir haben deutlich gemacht, dass wir etwas dafür tun müssen, um diejenigen, die dauerhaft bei uns bleiben, besser zu integrieren. Auch die große Koalition hat sich mit dem Thema Integration befasst, aber um ganz ehrlich zu sein: Herausgekommen im Sinne des Rechts ist dabei nicht viel. Wenn die Bundeskanzlerin zu einem Integrationsgipfel einlädt, aber ihr Innenminister festlegt, dass dort nicht über das Aufenthaltsrecht gesprochen wird, dann ist klar, dass es eher um weiche Themen geht. Wichtig war aber auch, dass die Teilnehmer eine Vorbild- funktion für den Dialog über eine gelungene Integration gegeben haben.

Ich meine aber, die Bundespolitik ist dafür verantwortlich, die gesetzlichen Rahmenbedingungen so zu setzen, dass Integration auch wirklich gelingen kann. Und dafür müssen wir auch in Zukunft eine Menge tun. Ich meine, wir sollten zum Beispiel im Staatsbürgerschaftsrecht die sogenannte Optionslösung abschaffen. Junge Migrantinnen und Migranten sollen nicht länger gezwungen werden, sich zwischen dem deutschen Pass und der Staatsangehörigkeit ihrer Eltern zu entscheiden. Wer nur Deutscher auf Zeit ist, wer mit spätestens 23 Jahren seinen deutschen Pass vielleicht wieder verliert, der wird sich hier nicht wirklich heimisch fühlen. Wir sollten deshalb bereit sein, doppelte Staatsangehörigkeiten häufiger hinzunehmen und das Optionsmodell abschaffen. Auch am Aufenthaltsrecht müssen wir weiter arbeiten - vor allem im Interesse der Frauen. Es darf nicht sein, dass Frauen, die Deutschland verlassen, schon nach sechs Monaten ihren Aufenthaltsstatus verlieren. Wir alle wissen von den Fällen: Junge Frauen, die in Deutschland aufgewachsen sind, werden im Heimatland ihrer Eltern zwangsweise verheiratet. Wenn diese Frauen aus solch einer Ehe ausbrechen und zurück nach Deutschland wollen, dann dürfen unsere Gesetze das nicht verhindern. Seinen Lebenspartner selbstbestimmt zu wählen, das ist nämlich auch ein Menschenrecht. Ich meine deshalb: Für diese Fälle der Heiratsverschleppung brauchen wir eine Sonderregelung im Aufenthaltsrecht. 


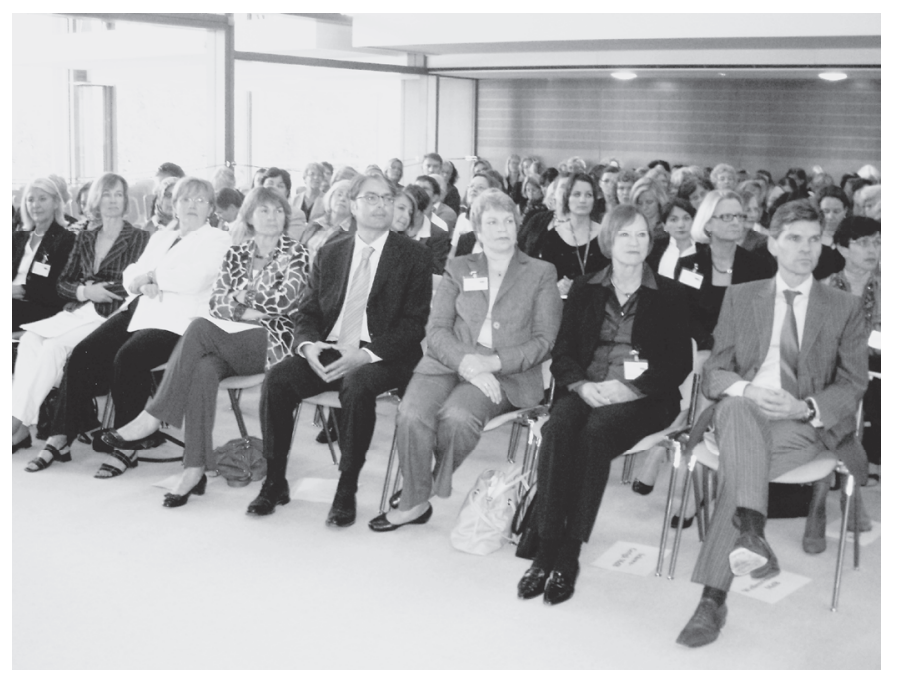

$\Delta \quad$ In der ersten Reihe (v.r.n.I.): Ingo Wellenreuther MdB, Irmingard ScheweGerigk MdB, Sibylle Laurischk MdB, Johannes Jung MdB, Präsidentin des BAG Ingrid Schmidt, Richterin am EGMR Dr. h.c. Renate Jaeger, Generalanwältin am EuGH Prof. Dr. Dr. Juliane Kokott, Präsidentin des Landesverfassungsgerichtshofs Berlin Margret Diwell (Foto: $C D$ )

Meine Damen und Herren,

Integration funktioniert besonders gut durch den Beruf, also am Arbeitsplatz. Aus diesem Grund ist auch die Anerkennung ausländischer Bildungsabschlüsse ein ganz wichtiger Aspekt der Integration. Viele Zuwanderer sind in ihrer Heimat gut ausgebildet worden und müssen dann hier erleben, dass ihre Qualifikationen nicht anerkannt werden. Damit lassen wir nicht nur ein enormes Potential ungenutzt, sondern wir sorgen auch für Frust bei den Zuwanderern und erschweren die Integration. Wir brauchen deshalb eine bessere Anerkennung der Abschlüsse und eine solide rechtliche Grundlage hierfür. Wir brauchen auch mehr Teilhabe für diejenigen, die keinen deutschen Pass haben. Warum lassen wir zwar EU-Bürgerinnen in den Kommunen wählen, aber nicht die rund vier Millionen Menschen aus der Türkei, Serbien oder etwa Kroatien, die dauerhaft bei uns leben? Dies sind nur drei Beispiele dafür, wie gesetzliche Regelungen helfen können, dass Menschen unterschiedlicher Herkunft glücklich und friedlich zusammenleben. Ich bin mir sicher, dass der djb und dieser Kongress noch weitere Anregungen geben, wie wir das erreichen können, und deshalb bin ich auf Ihre rechtspolitischen Empfehlungen in dieser Sache auch sehr gespannt.

Meine Damen und Herren,

lassen sie mich noch ein Wort sagen zu einem ganz anderen Thema, das dem djb und mir ebenfalls sehr am Herzen liegt: Es geht um Frauen in den Aufsichtsräten und in den Vorständen deutscher Unternehmen. In der vergangenen Woche hat hier in dieser Stadt das Karlsruhe Institute of Technology eine bemerkenswerte Studie vorgestellt. Sie untersucht erstmals die 600 führenden Unternehmen in Deutschland. Die Zahlen, die dabei herausgekommen sind, sind frustrierend: Von 1721 Vorständen sind ganze 42 Frauen. Das sind 2,4 Prozent. Bei den Aufsichtsräten sind es gut acht Prozent. Nennenswerte positive Veränderungen hat es in den letzten zehn Jahren nicht gegeben. Im Gegenteil, seit 2005 ist der Frauenanteil sogar wieder leicht rückläufig. Der Deutsche Juristinnenbund und auch ich setzen uns seit geraumer Zeit dafür ein, dass endlich Schluss ist mit der reinen Männerwirtschaft in den Führungsetagen deutscher Unternehmen. Angesichts dieser neuen Zahlen wird für mich aber eines sehr deutlich: Appelle, Förderprogramme und guter Wille allein reichen nicht aus. Ganz offenkundig ist auch hier eine „Integration durch Recht“ erforderlich. Ich meine, es war deshalb richtig, was meine Partei in ihrem Regierungsprogramm festgeschrieben hat: Wir wollen nach dem Vorbild anderer Länder eine Frauenquote für Aufsichtsräte schaffen. Dieses Ziel zu erreichen, wird gewiss nicht einfach weder politisch noch juristisch. Trotzdem werden wir es politisch angehen - Jutta Wagner hat recht: Die Zeit ist reif dafür.

Bei diesem und vielen anderen Vorhaben biete ich dem Deutschen Juristinnenbund auch künftig eine enge Zusammenarbeit an. Der djb ist und bleibt für das Bundesjustizministerium ein ganz wichtiger Partner. Deshalb wünsche ich Ihnen weiterhin eine erfolgreiche Arbeit und einen erfolgreichen Kongress hier in Karlsruhe.

\section{Grundrechte ziehen klare Grenzen und eröffnen Räume der Teilhabe}

\author{
Prof. Dr. Ulrich Goll MdL (FDP) \\ Justizminister und Integrationsbeauftragter der Landesregie- \\ rung Baden-Württemberg, Stuttgart
}

Sehr geehrte Frau Präsidentin Wagner, sehr geehrter Herr Bundesverfassungsgerichtspräsident Professor Papier, sehr geehrte
Frau Kollegin Zypries, sehr geehrte Frau Professor LübbeWolff, sehr geehrte Damen und Herren,

im Namen der Landesregierung und auch persönlich begrüße ich Sie alle hier in Karlsruhe sehr herzlich zum 38. Kongress des Deutschen Juristinnenbundes. Angesichts der Tatsache, dass von den rund 15 Millionen in Deutschland lebenden 